TRANSACTIONS OF THE

AMERICAN MATHEMATICAL SOCIETY

Volume 360, Number 4, April 2008, Pages 2191-2204

S 0002-9947(07)04374-7

Article electronically published on November 28, 2007

\title{
FREE LINES FOR HOMEOMORPHISMS OF THE OPEN ANNULUS
}

\author{
LUCIEN GUILLOU
}

\begin{abstract}
Let $H$ be a homeomorphism of the open annulus $S^{1} \times \mathbf{R}$ isotopic to the identity and let $h$ be a lift of $H$ to the universal cover $\mathbf{R} \times \mathbf{R}$ without fixed point. Then we show that $h$ admits a Brouwer line which is a lift of a properly imbedded line joining one end to the other in the annulus or $H$ admits a free essential simple closed curve.
\end{abstract}

\section{INTRODUCTION}

Our main result in this note is the following:

0.1. Theorem. Let $H$ be a homeomorphism of the open annulus $S^{1} \times \mathbf{R}$ isotopic to the identity which admits a lift $h$ to $\mathbf{R}^{2}$ without fixed point. Then there exists an essential simple closed curve in the annulus free under $H$ (and therefore it lifts to a Brouwer line for $h$ ) or there exists a properly imbedded line in the annulus joining both ends which lifts to a Brouwer line for $h$.

A natural question, in case $H$ is fixed point free, is to ask, in the second case, for a properly imbedded line in the annulus joining both ends and free for $H$. It has recently been given a positive answer in many cases by F. Béguin, S. Crovisier and F. Le Roux [3].

Note. A slightly weaker form of this theorem was communicated to a few people beginning in 1994 but not published partly because I was unable to answer the preceding question. In his 2000 thesis (directed by Le Calvez), Sauzet [10] reproved in his own way the exact statement above, but this thesis also stayed unpublished. Recently, Addas-Zanata [1] and Béguin-Crovisier-Le Roux [3] used this result as a step towards interesting dynamical results, so we decided to publish these notes. Our original proof relied on hard to read original work of Kérékjartó [8]; we tried here to give a proof starting from first principles. The main novelty of our approach lies in the finiteness property of Lemma 3.6, which leads quickly to various periodicity properties, at least on surfaces with abelian fundamental group. The approach with free sets (as the bricks of [10]), instead of critical sets as here, needs more work to be complete, particularly a study of an abstract dynamical system on the set of bricks associated to $h$. Notice however that this last approach presently seems the right way to get general periodicity results. Indeed as remarked in [3], Theorem 0.1 , for the case of area preserving homeomorphisms, follows quickly from

Received by the editors June 14, 2006.

2000 Mathematics Subject Classification. Primary 37E30.

Key words and phrases. Brouwer homeomorphism, free line, fixed point, open annulus, torus, Poincaré-Birkhoff Theorem. 
the much harder recent "version feuilletée équivariante du théorème de Brouwer" of Le Calvez [9].

For comparison recall that in [6] we proved that if $H$ is a fixed point free orientation preserving homeomorphism of the closed annulus, then there exists an essential simple closed curve in the annulus free under $H$ or there exists an essential simple arc in the annulus free under $H$ generalizing the celebrated Poincaré-Birkhoff theorem. Part of the arguments given here can easily be adapted to prove this result too. Instead, these arguments will be used to prove

0.2. Theorem. Let $H$ be a homeomorphism of the torus $\mathbf{T}^{2}$ preserving orientation which admits a lift to $\mathbf{R}^{2}$ without fixed point. Then there exists an essential simple closed curve on $\mathbf{T}^{2}$ which lifts to a Brouwer line of $h$.

Note that in the case of the torus, we cannot ask for an essential simple curve in $\mathbf{T}^{2}$ free under $H$ : Bestvina and Handel have given an example of a fixed point free, homotopic to the identity, homeomorphism of the torus without any free essential simple closed curve [4].

Finally, in section 5, we show how Theorem 0.1 can be used to solve a conjecture of Winkelnkemper about area preserving homeomorphisms of the 2-sphere with exactly two stable fixed points which generalises the classical Poincaré-Birkhoff Theorem on the closed annulus.

\section{BASIC FACTS ABOUt BrouWER homeOMORPHisms}

In this paragraph $h$ will be a fixed point free orientation preserving homeomorphism of the plane $\mathbf{R}^{2}$. Such a homeomorphism is called a Brouwer homeomorphism.

The most basic fact about these homeomorphisms is that they automatically have no periodic points or even recurrent points. More generally we have the following lemma of J. Franks [5] for which we recall the following definition.

1.1. Definition. Let $f$ be a homeomorphism of $\mathbf{R}^{2}$. A subset $E$ of $\mathbf{R}^{2}$ is free (for $f)$ if $E \cap f(E)=\emptyset$. It is critical if int $E$ is free but $f(E) \cap E \neq \emptyset$.

1.2. Lemma. Let $\left(B_{i}\right)_{i=1, \ldots, k}(k \geq 1)$ be a sequence of open discs in $\mathbf{R}^{2}$ and $f$ an orientation preserving homeomorphism of $\mathbf{R}^{2}$ such that:

1. $B_{i} \cap B_{j}=\emptyset$ for all $i \neq j, 1 \leq i, j \leq k$;

2. each $B_{i}$ is free for $f$ for each $1 \leq i \leq k$;

3. there exist integers $n_{i}>0$ such that $f^{n_{i}}\left(B_{i}\right) \cap B_{i+1} \neq \emptyset$ for each $i \in \mathbf{N}$ where $i$ is taken $\bmod k$.

Then there exists a simple closed curve of index 1 for $f$ and, therefore, a fixed point for $f$.

We now fix a Brouwer homeomorphism $h$. As a consequence of Franks' Lemma, every point in the plane is wandering under $h$.

1.3. Definition. A simple closed arc $\alpha \subset \mathbf{R}^{2}$ with endpoints $p$ and $h(p)$ for some $p \in \mathbf{R}^{2}$ is a translation arc if $\alpha \cap h(\alpha)=\{h(p)\}$. A translation arc will always be supposed to be oriented from $p$ to $h(p)$.

1.4. Proposition. Every point of $\mathbf{R}^{2}$ is contained in a translation arc. If $\alpha$ is a translation arc, the trajectory $\bigcup_{n \in \mathbf{Z}} h^{n}(\alpha)$ is a submanifold of $\mathbf{R}^{2}$ homeomorphic to $\mathbf{R}$. 
Such a trajectory $l$ is not necessarily closed as a subset of $\mathbf{R}^{2}$ but, being a submanifold, $\bar{l} \backslash l$ is closed and we have:

1.5. Proposition. A trajectory $l$ divides the connected component of $\mathbf{R}^{2} \backslash(\bar{l} \backslash l)$ containing $l$ into exactly two components.

We refer to [6] for a proof of the two preceding propositions.

\section{Critical Discs and Brouwer Plane translation theorem}

The content of this section may be seen as a variation on [11].

Again let $h$ be a Brouwer homeomorphism.

A disc will always be a closed disc in that paper; otherwise we say "open disc".

2.1. Lemma. If the disk $D$ is free or critical, then $D \cap h^{n}(D)=\emptyset$ for every $n \in \mathbf{Z} \backslash\{0, \pm 1\}$.

Proof. Let us suppose by contradiction that there exists a point $x \in D$ such that $h^{n}(x) \in D$ for some $n>1$. Any arc from $x$ to $h^{n}(x)$ contained in int $D$ except, eventually, for its endpoints, would be free since $h$ has no periodic point. Then, one could find an open disc, that is a neighborhood of this arc, which contradicts Franks' Lemma (with $i=1$ ).

2.2. Lemma. Let $D$ be a critical disc. Then the boundary of $D$ decomposes into two disjoint arcs (eventually degenerate, that is, reduced to a point) containing respectively $h^{-1}(D) \cap D$ and $D \cap h(D)$ and two translations arcs, these four arcs having disjoint interiors.

Proof. Because of Jordan's theorem, $h^{-1}(D) \cap D$ and $D \cap h(D)$ are not linked on $\partial D$ (that is, are contained in disjoint intervals of $\partial D$ ). Therefore there exists a smallest closed sub-arc $\lambda$ (eventually reduced to a point) of $\partial D$ containing $h^{-1}(D) \cap D$ and disjoint from $h(D)$. According to the preceding lemma and Jordan's Theorem, $h(D) \cap D$ is contained either in $\operatorname{int} \lambda$ or $\partial D \backslash \lambda$. In the first case, the frontier of the unbounded component of $\mathbf{R}^{2} \backslash\left(h^{-1}(D) \cup D\right)$ is a Jordan curve and is sent into the disc it bounds by $h$, which gives a fixed point for $h$, a contradiction. Therefore, the second case holds and the arc $\mu$, which is the smallest closed sub-arc (eventually reduced to a point) of $\partial D$ containing $D \cap h(D)$, is disjoint from $\lambda$. The end points of $\mu$ and $h(\lambda)$ coincide and, since $h$ preserves orientation, the two sub-arcs of $\partial D$ which are the components of $\partial D \backslash(\operatorname{int} \lambda \cup \operatorname{int} \mu)$ are translation arcs.

2.3. Definition. A translation arc as in the preceding lemma, oriented as said before, will be said to be high if $D$ is on the right of the translation arc and low if $D$ is on the left of the translation arc. It will be denoted by $\alpha_{+}$or $\alpha_{-}$respectively.

CONVENTION. From now on, unless said otherwise, all discs will be Euclidean discs.

2.4. Definition. Let us consider two critical discs $D^{\prime}$ and $D$ which satisfy:

1. $\partial D \cap D^{\prime}$ is a nondegenerate sub-arc of a translation arc $\alpha$ of $\partial D$ (which means contained in $\partial D)$.

2. $h^{-1}(D) \cap \operatorname{int} D^{\prime}=\emptyset=\operatorname{int} D^{\prime} \cap h(D)$.

$D^{\prime}$ will be called an extension of $D$ (high or low according to the type of the $\operatorname{arc} \alpha)$. 
2.5. Remarks. 1. $D^{\prime}$ is a high extension of $D$ if and only if $D$ is a low extension of $D^{\prime}$. Indeed, it is enough to check that the low translation arc of $D^{\prime}$ contains $\partial D^{\prime} \cap D$. But, if this were not true, int $D$ would contain a point of $D^{\prime} \cap h\left(D^{\prime}\right)$ or of $D^{\prime} \cap h^{-1}\left(D^{\prime}\right)$ in contradiction to point 2 of the definition.

2. $D \cup D^{\prime}$ is still a critical topological disc when $D^{\prime}$ is an extension of $D$.

2.6. Lemma. Let $D$ and $D^{\prime}$ be two critical discs and $\alpha_{-}, \alpha_{+}$the two arcs of translations of $\partial D$. Suppose that int $D^{\prime} \cap \partial D$ is not empty, meets $\alpha_{+}$and that $D^{\prime} \cap\left(h^{-1}\left(\alpha_{+}\right) \cup h\left(\alpha_{+}\right)\right)=\emptyset$. Then, $D \cup D^{\prime}$ is a critical topological disc and $D^{\prime}$ is a high extension of $D$.

2.7. Remark. Of course, we have an analogous lemma obtained by exchanging $\alpha_{+}$ and $\alpha_{-}$and "high" and "low".

Proof. We will first prove

2.8. Assertion. Let $D$ be any critical or free topological disc and $\alpha$ any translation arc such that int $D \cap \alpha \neq \emptyset$ and $D \cap\left(h^{-1}(\alpha) \cup h(\alpha)\right)=\emptyset$. Then $D \cap \bar{l}=D \cap \alpha$ where $l$ is the trajectory generated by $\alpha$.

Given this assertion (applied to $D^{\prime}$ and $\alpha_{+}$) and the fact that $l$ separates the connected component of $\mathbf{R}^{2} \backslash(\bar{l} \backslash l)$ containing $l$ into two components invariant under $h$, one concludes easily the proof of the lemma (recall that $D$ and $D^{\prime}$ are Euclidean discs here).

Proof of the Assertion. Suppose first that $D \cap h^{n}(\alpha) \neq \emptyset$ for some $n \in \mathbf{Z},|n|>1$. We can find an arc $\gamma$ in int $D$, except perhaps for one end point, joining a point $x \in \operatorname{int} \alpha \cap \operatorname{int} D$ to a point $h^{n}(y)$ for some $y \in \alpha$. Since $\operatorname{int} D$ and the subarc $[x, y]$ of $\alpha$ are free, $\gamma$ is free also. Then, small enough open topological discs, neighborhoods of $[h(x), h(y)]$ and $\gamma$ respectively, would satisfy the hypothesis of Franks' Lemma giving rise to a fixed point for $h$ and a contradiction.

If $D \cap \bar{l} \neq \emptyset$, we can suppose that $D \cap l=\emptyset$ from what precedes and we can find a free $\operatorname{arc} \gamma$ inside $\operatorname{int} D$, except perhaps for one end point, joining a point $x \in \operatorname{int} \alpha \cap \operatorname{int} D$ to a point $z \in \bar{l} \backslash l$. We then choose a small free disc $D^{\prime}$ around $z$ such that $D^{\prime} \cap\left(h^{-1}(\gamma) \cup h(\gamma)\right)=\emptyset$. Then $D^{\prime} \cup \gamma$ is free and there exists a free topological disc that is a small enough neighborhood of $D^{\prime} \cup \gamma$ and that does not meet $h^{-1}(\alpha) \cup h(\alpha)$ but meets $\alpha$ and $h^{n}(\alpha)$ for some $n \in \mathbf{Z},|n|>1$, as $D$ in the first paragraph, which leads again to a contradiction.

2.9. Lemma. If $\left(D_{i}\right)_{i \in I}$ (where $I$ is a finite set $\{0,1, \ldots, n\}$ or $\mathbf{N}$ or $\mathbf{Z}$ ) is a sequence of critical discs such that $D_{i+1}$ is a high extension of $D_{i}$, then $\bigcup_{i \in I} D_{i}$ is a critical disc. Furthermore, $\left(D_{i} \backslash D_{i-1}\right) \cap\left(D_{j} \backslash D_{j-1}\right)=\emptyset$ if $i \neq j$.

Proof. Let $l_{i}$ be the translation line generated by the high translation $\operatorname{arc}$ of $D_{i}$. By Assertion 2.8, the high translation arc of $D_{i+1}$ does not meet $\bar{l}_{i}$ and we define $\Sigma_{i}$ as the component of $\mathbf{R}^{2} \backslash\left(\bar{l}_{i} \backslash l_{i}\right)$ which contains the high translation arc of $D_{i+1}$. It follows from the preceding lemma that $\Sigma_{i} \supset \Sigma_{i+1}$. Using the invariance of these $\Sigma_{i}$ under $h$ one gets the lemma by an easy induction (and, in case $I=\mathbf{Z}$, a second induction going from $i$ to $i-1$ and using the low translation $\operatorname{arc}$ of $D_{i}$ ).

To each point $x$ in the plane, we now associate the radius $r_{x}$ of the Euclidean critical disc with center at $x$.

2.10. Lemma. The map $x \mapsto r_{x}$ is continuous. 
Proof. This follows from the fact that, if $d(x, y)<\epsilon$, then $B\left(y, r_{x}-\epsilon\right) \subset B\left(x, r_{x}\right) \subset$ $B\left(y, r_{x}+\epsilon\right)$.

2.11. Lemma. Let $D$ be a critical disc and $\alpha$ be one of the two translation arcs of $\partial D$. There exists a critical disc $D^{\prime}$ with center on $\alpha$ such that $D^{\prime}$ is an extension (high or low according to the choice of $\alpha$ ) of $D$.

Proof. Let $C_{+}$(resp. $C_{-}$) be the set of points $x$ in $\alpha$ such that the critical disc with center at $x$ meets $h(\operatorname{int} D)$ (resp. $\left.h^{-1}(\operatorname{int} D)\right)$. To prove the lemma it is enough to prove that $C_{+} \cup C_{-}$is not all of $\alpha$. This will follow from the connectivity of $\alpha$ and the following three points.

- $C_{+} \cap C_{-}=\emptyset$ : Let $D^{\prime}$ be a Euclidean critical disc in $C_{+} \cap C_{-}$, that is, meeting $h(\operatorname{int} D)$ and $h^{-1}(\operatorname{int} D)$. Then there exists a topological $\operatorname{disc} \Delta \subset \operatorname{int} D^{\prime} \backslash \operatorname{int} D$ meeting both $h(\operatorname{int} D)$ and $h^{-1}(\operatorname{int} D)$ which gives with $\operatorname{int} D$ two $\operatorname{discs}$ satisfying the hypothesis of Franks' Lemma and therefore a contradiction.

- $C_{+}$and $C_{-}$are not empty: it is enough to consider discs centered on the end points of $\alpha$.

- $C_{+}$and $C_{-}$are open: this follows from the continuity of the map $x \mapsto r_{x}$.

Under the hypothesis and notation of the preceding lemma, since $D^{\prime}$ is critical, one can repeat the construction using this time the translation arc on $\partial D^{\prime}$ of the same type as $\alpha$. One gets in this way an infinite sequence $D_{0}=D, D_{1}=$ $D^{\prime}, D_{2}, \ldots, D_{n}, \ldots$ of critical discs such that by Lemma 2.9 , for every $n, \bigcup_{i=0}^{i=n} D_{i}$ is a critical disc and $\left(D_{i} \backslash D_{i-1}\right) \cap\left(D_{j} \backslash D_{j-1}\right)=\emptyset$ if $i \neq j$.

2.12. Lemma. In this situation, the sequence $\left\{D_{n}\right\}$ converges to infinity, that is, given any compactum $K \subset \mathbf{R}^{2}$, there exists $N$ such that the connected set $\bigcup_{i \geq N} D_{i}$ does not meet $K$.

Proof. Since the $D_{n}$ are critical, the radius of those meeting $K$ is bounded below as well as their area. By Lemma 2.9, all $D_{n} \backslash D_{n-1}$ are disjoint and, by Euclidean geometry, have area greater than half the area of $D_{n}$. Therefore only a finite number of discs $D_{n}$ can meet $K$.

It is now easy to prove the Brouwer plane translation theorem.

2.13. Definition. A Brouwer line $L$ for the Brouwer homeomorphism $h$ is the image of a proper embedding of $\mathbf{R}$ into $\mathbf{R}^{2}$ such that

1. $L$ is free under $h$,

2. $L$ separates $h(L)$ and $h^{-1}(L)$.

2.14. Plane translation theorem (Brouwer). Let $h$ be a Brouwer homeomorphism. Then every point in $\mathbf{R}^{2}$ belongs to a Brouwer line.

Proof. Let $x$ be the point in consideration and $D_{0}$ the critical disc centered at $x$. One constructs as above a sequence $D_{0}, D_{1}, \ldots, D_{n}, \ldots$ of critical discs converging to infinity, and also, starting from the other translation arc of $D_{0}$, another sequence $D_{0}, D_{-1}, \ldots, D_{-n}, \ldots$ of critical discs converging to infinity. Note that $\bigcup_{k=i}^{k=j} D_{k}$ is critical for $i<j$ where $i, j \in \mathbf{Z}$ since one can see that finite sequence as obtained by the preceding construction from $D_{i}$ and therefore $\bigcup_{i \in \mathbf{Z}} \operatorname{int} D_{i}$ is free. It is then easy to construct inside $\bigcup_{i \in \mathbf{Z}} \operatorname{int} D_{i}$ a proper embedding $L$ of $\mathbf{R}$ joining linearly the 
centers of the $D_{i}$ 's. It has to be free and also has to satisfy property 2 of a Brouwer line since one can find in $D_{0}$ a simple arc joining $h^{-1}\left(D_{0}\right)$ to $h\left(D_{0}\right)$ crossing $L$ only at $x$ and if $L$ were not separating $h^{-1}(L)$ and $h(L)$, one could complete this arc into a simple closed curve crossing $L$ only at $x$ in contradiction to the properness of $L$.

\section{Further Results on EuClidean CRITICAl Discs}

The goal of this section is to obtain the finiteness property of Lemma 3.6. It will follow from an easy compactness argument once the notions of critical disc and of extension are suitably modified so that being an extension becomes an open property.

We still fix a Brouwer homeomorphism $h$.

3.1. Definition. Let $D$ be a critical disc. We know that its boundary is the union of two translation arcs $\alpha_{+}$and $\alpha_{-}$(high and low respectively) and of two disjoints arcs, $\lambda$ and $\mu$, which are the smallest sub-arcs of $\partial D$ containing $h^{-1}(D) \cap D$ and $D \cap h(D)$ respectively. Choose $\epsilon$ so that $0<\epsilon \leq \frac{1}{8} \inf \left(l\left(\alpha_{+}\right), l\left(\alpha_{-}\right), l(\lambda), l(\mu)\right)$ where $l($.$) is the length of the corresponding arc and where we omit l(\lambda)$ and $l(\mu)$ if $\lambda$ (or equivalently $\mu$ ) is degenerate. We have $\epsilon \leq \frac{\pi}{8} r$ if $r$ is the radius of $D$.

Then $D$ without the discs of radius $\epsilon$ centered at the end points of $\alpha_{-}$is a topological disc $D_{+}$, and $D$ without the discs of radius $\epsilon$ centered at the end points of $\alpha_{+}$is also a topological disc $D_{-}$. If $\lambda\left(\right.$ or $\mu$ ) is not degenerate, then $D_{+}$and $D_{-}$ are topological critical discs and otherwise $D_{+}=D_{-}$is a free topological disc. In any case, $\operatorname{area}\left(D_{ \pm}\right)>\frac{2 \pi}{3} r^{2}$.

So, to each point $x$ of the plane we may associate three critical topological discs: the Euclidean critical disc $D$ centered at $x$ and two derived topological discs $D_{+}$ and $D_{-}$. These three discs will be called generalized critical discs. The centers, radii and translation arcs of the derived discs are those of $D$ by definition. We will extend this vocabulary to the case where $\lambda$ and $\mu$ are degenerate: in that case $D_{+}=D_{-}$is free and not critical but, having its area bounded below as said above, it will not cause any problem below.

The Euclidean critical disc $D$ will be called the underlying disc of $D_{+}$and $D_{-}$and also $D$ on occasion.

We will begin to improve the extension Lemma 2.11 as follows.

3.2. Lemma. Let $D$ be a critical disc and $\alpha$ one of the two translation arcs of $\partial D$. Then there exists a generalised critical disc $D^{\prime}$ such that

1. the underlying disc of $D^{\prime}$ is an extension of $D$,

2. $D^{\prime} \cap \partial D=D^{\prime} \cap i n t \alpha$,

3. $h^{-1}(D) \cap D^{\prime}=\emptyset=D^{\prime} \cap h(D)$.

3.3. Definition. Given two critical discs $D_{1}$ and $D_{2}$ admitting derived discs $D$ and $D^{\prime}$ which satisfy conditions 1,2 and 3 above we will say that $D_{2}$ is a strict extension of $D_{1}$ (high or low according to the type of $\alpha$ ).

3.4. Remarks. 1. $D \cup D^{\prime}$ is a topological critical disc (or free if $D$ and $D^{\prime}$ are free).

2. If $D_{2}$ is a strict high (resp. low) extension of $D_{1}$, then $D_{1}$ is a strict low (resp. high) extension of $D_{2}$.

3. Being a strict extension is an open condition. 
Proof of Lemma 3.2. Let $A_{+}$(resp. $A_{-}$) be the subset of $\alpha$ of points $x \in \alpha$ such that the critical disc with center at $x$ meets $h(D)\left(\right.$ resp. $\left.h^{-1}(D)\right)$. Then

- $A_{+}$and $A_{-}$are not empty: it is enough to look at critical discs centered at the end points of $\alpha$.

- $A_{+}$and $A_{-}$are closed: this follows from the continuity of the map $x \mapsto r_{x}$.

- If $A_{+} \cap A_{-}=\emptyset$, the connectivity of $\alpha$ implies that an honest critical disc satisfies the lemma.

- If $A_{+} \cap A_{-} \neq \emptyset$, then this intersection is reduced to only one disc: the one centered at the midpoint of $\alpha$ and with the end points of $\alpha$ on its boundary.

Indeed, if there were a disc $D^{\prime}$ meeting $h^{-1}(D), h(D)$ and at most one end point of $\alpha$, one could find a free arc $\delta$ contained in $D^{\prime} \backslash D$ except for its end points $a \in h^{-1}(D)$ and $b \in h(D)$. One could also find a free $\operatorname{arc} \gamma$ in $D$, disjoint from $\delta$ and joining $h(a)$ to $h^{-1}(b)$ and therefore, by fattening, a contradiction with Franks' Lemma.

Note that the same argument shows that a critical disc $D^{\prime}$ which contains the two end points of $\alpha$ cannot meet $h^{-1}(D)$ or $h(D)$ except in these end points.

Then that one disc $D^{\prime}$ admits a derived disc $\left(D_{+}^{\prime}\right.$ or $D_{-}^{\prime}$ according to the type of $\alpha$ ) which satisfies the first case of the lemma.

3.5. Lemma. Analogs of Lemmas 2.9 and 2.12 are now equally true with extension replaced by strict extension and with the same proofs (for the second lemma we use the fact that the free discs eventually met have an area bounded below as said above).

It is only to get the following lemma that we had to consider strict extensions and derived discs. But of course this lemma will be crucial in section 4 .

3.6. Lemma. Let $K$ be a compactum in the plane. There exists a finite number $n$ of pairs of generalised discs $\left(D_{i}^{1}, D_{i}^{2}\right), 1 \leq i \leq n$, such that every critical disc centered on $K$ admits one of these pairs as a couple of strict extensions: high and low.

Proof. If $D$ is a Euclidean critical disc centered at $x$ and $\left(D^{1}, D^{2}\right)$ a pair of Euclidean generalised critical discs such that $D^{1}$, is a strict high extension of $D$ and $D^{2}$ is a strict low extension of $D$, then there exists a neighborhood $V_{x}$ of $x$ such that the Euclidean critical discs centered on $V_{x}$ all admit $D^{1}$ and $D^{2}$ as strict high and low extensions (since $x \mapsto r_{x}$ is continuous and the $\operatorname{arcs} \partial D \cap D^{1}$ and $\partial D \cap D^{2}$ being free, neighboring arcs are still free). A finite subcovering of the covering of $K$ by the $V_{x}$ 's leads to the sought after pairs.

\section{HomeOmorphisms of THE TORUS AND THE OPEN ANNUluS}

We continue to call $h$ a Brouwer homeomorphism of the plane.

We will denote by $s$ and $t$ the applications $(x, y) \mapsto(x, y+1)$ and $(x, y) \mapsto$ $(x+1, y)$ of $\mathbf{R}^{2}$. Also, $\pi$ will be the projection $\mathbf{R}^{2} \rightarrow \mathbf{R} / \mathbf{Z} \times \mathbf{R}$.

4.1. Definition. If $L$ is a Brouwer line for $h$, we denote by $D(L)$ the component of $\mathbf{R}^{2} \backslash L$ which contains $h(L)$ and by $G(L)$ the one which contains $h^{-1}(L)$.

4.2. Lemma. Let $\left(L_{n}\right)_{n \in \mathbf{Z}}$ be a locally finite family of Brouwer lines for $h$ such that $\overline{\bigcup_{n} D\left(L_{n}\right)}$ is connected and consider an unbounded component $R$ of $\mathbf{R}^{2} \backslash \overline{\bigcup_{n} D\left(L_{n}\right)}$. Then, the frontier of $R$ is a Brouwer line for $h$. 
Proof. Given the local finiteness of the family, $\overline{\bigcup_{n} D\left(L_{n}\right)}=\bigcup_{n} \overline{D\left(L_{n}\right)}$ and a classical result of Kérékjartó [7, page 87] implies that $\operatorname{Fr} R$ is a submanifold of the plane. $\mathbf{R}^{2}$ being unicoherent (that is, if it is represented as the union of two closed connected sets, the intersection of these sets is connected) and $\overline{\bigcup_{n} D\left(L_{n}\right)}$ connected, Fr $R$ is connected [13, exercise 9, pp. 113 and 117]. But the frontier of $R$ is free, because if $x \in \operatorname{Fr} R$, there is $n$ such that $x \in L_{n}$ and therefore, $h(x) \in D\left(L_{n}\right) \subset \mathbf{R}^{2} \backslash \bar{R}$. Since $h^{-1}(R) \subset R$ and $h$ has no fixed point, it cannot be a circle and so it is a proper free line. It is a Brouwer line since $h^{-1}(R) \subset R$ and $h(R) \subset \mathbf{R}^{2} \backslash \bar{R}$.

4.3. Lemma. Suppose $h$ commutes with $t$ and let be given a Brouwer line $L$ for $h$ which projects onto and properly on the y-axis. Then there exists a Brouwer line $L^{\prime}$ for $h$ which projects onto and properly on the $y$-axis and is free for $t$.

Furthermore, if $h$ commutes also with $s$ and if $L$ is invariant under $s$, one can find an $L^{\prime}$ that is also invariant under $s$.

Proof. Since $L$ projects down properly onto the $y$-axis, there exists $N>0$ such that $G(L)$ or $D(L)$ contains $[N,+\infty) \times\{0\}$. Exchanging $h$ and $h^{-1}$ we can suppose that $D(L)$ contains a half line $[N,+\infty) \times\{0\}$ and consider the unbounded connected component $R$ of $\mathbf{R}^{2} \backslash \overline{\bigcup_{k \geq 0} t^{k}(D(L))}$ and its frontier $L^{\prime}$.

For every $A>0$ there exists $n(A)$ such that for $n \geq n(A), t^{n}(L \cap \mathbf{R} \times[-A, A]) \cap$ $\mathbf{R} \times[-A, A]=\emptyset$. Therefore the family $\left(t^{k}(L)\right)_{k \geq 0}$ is locally finite and $L^{\prime}$ projects onto and properly on the $y$-axis. Furthermore, by Lemma $4.2, L^{\prime}$ is a Brouwer line and it satisfies $t\left(L^{\prime}\right) \subset \bar{D}\left(L^{\prime}\right)$ since if $x \in L^{\prime}$, there exists $n$ such that $x \in t^{n}(L)$ and so $t(x) \in t^{n+1}(\bar{D}(L)) \subset \mathbf{R}^{2} \backslash R=\bar{D}\left(L^{\prime}\right)$.

Notice that, since $s$ and $t$ commute, if $s(L)=L$, then also $s\left(L^{\prime}\right)=L^{\prime}$.

Therefore, to conclude that there exists a Brouwer line going down in the annulus on a free line joining the two ends, it is enough to apply the following sublemma to $L^{\prime}$.

4.4. Sublemma. Let $L$ be a Brouwer line for $h$ with a proper onto projection on the $y$-axis such that $t(L) \subset \bar{D}(L)$. Then there exists a Brouwer line $L^{\prime}$ with a proper onto projection on the $y$-axis such that $t\left(L^{\prime}\right) \subset D\left(L^{\prime}\right)$.

Furthermore, if $h$ commutes with $s$ and $s(L)=L$, then one can choose $L^{\prime}$ such that $s\left(L^{\prime}\right)=L^{\prime}$.

Proof. Let $\delta: L \rightarrow] 0,+\infty$ [ be a function such that the $\delta$-neighborhood $W$ of $L$ does not meet $h^{-1}(W) \cup h(W)$ and define $L_{0}=\emptyset$ and, for all $k>0, L_{k}=L \cap(\mathbf{R} \times[-k, k])$. Since $L_{k}$ is compact, there exists $n=n(k)$ such that $L_{k} \cap t^{n}\left(L_{k}\right)=\emptyset$. Let us suppose that $L_{k-1} \cap t\left(L_{k-1}\right)=\emptyset$ and $n>1$ and let us show that there exists a Brouwer line $L^{\prime}$ with a proper onto projection on the $y$-axis such that $L_{k-1}^{\prime}=L_{k-1}$, $t\left(L^{\prime}\right) \subset \bar{D}\left(L^{\prime}\right)$, and $L_{k}^{\prime} \cap t^{m}\left(L_{k}^{\prime}\right)=\emptyset$ for some $m, 0<m<n$. (This is enough to finish the proof of the sublemma.)

Define $X_{i}=L_{k} \cap \ldots \cap t^{i}\left(L_{k}\right)$ if $i \geq 0$. By hypothesis, $t\left(X_{n-1}\right) \cap L_{k}=X_{n}=\emptyset$ and $X_{n-1}$ is a compact of $L_{k}$ disjoint from $L_{k-1}$ as well as the $\delta$-neighborhood of $X_{n-1}$ (by diminishing $\delta$ near $X_{n-1}$ if necessary). The $\delta$-neighborhood of $X_{n-1}$ in $L$ is a finite union of open intervals in $L_{k+1} \backslash L_{k-1}$. Let us replace these intervals by arcs with the same end points but included in the intersection of $G(L)$ and of the $\delta$-neighborhood of $X_{n-1}$. We thus get a Brouwer line $L^{\prime}$ which projects onto and properly on the $y$-axis and such that $L_{k-1}^{\prime}=L_{k-1}$. We are left to check that $X_{n-1}^{\prime}=\emptyset$. 
To do that, notice first that since $L_{k}^{\prime} \subset\left(L_{k} \backslash X_{n-1}\right) \bigcup^{\hat{\alpha}}$ where $\hat{\alpha}$ is a union of arcs in $G(L)$ and $t(L) \subset \bar{D}(L)$, we have $t\left(L_{k}\right) \cap L_{k}^{\prime} \subset\left(t\left(L_{k}\right) \cap\left(L_{k} \backslash X_{n-1}\right)\right) \cup\left(t\left(L_{k}\right) \bigcap^{\hat{\alpha}}\right)$ $=\left(X_{1} \backslash X_{n-1}\right) \cup \emptyset=X_{1} \backslash X_{n-1}$. Also $t\left(L_{k}^{\prime}\right)$ and $t\left(L_{k}\right)$ differ only in the $\delta$ neighborhood of $t\left(X_{n-1}\right)$. For $\delta$ small enough, this neighborhood does not meet $L_{k}$ or $L_{k}^{\prime}$ and therefore $L_{k}^{\prime} \cap t\left(L_{k}^{\prime}\right)=L_{k}^{\prime} \cap t\left(L_{k}\right) \subset X_{1}=L_{k} \cap t\left(L_{k}\right)$. We deduce that $t^{i}\left(L_{k}^{\prime}\right) \cap t^{i+1}\left(L_{k}^{\prime}\right) \subset t^{i}\left(L_{k}\right) \cap t^{i+1}\left(L_{k}\right)$ for $i \geq 0$ and $X_{n-1}^{\prime} \subset X_{n-1}$ and conclude that $X_{n-1}^{\prime}=L_{k}^{\prime} \cap t\left(L_{k}^{\prime}\right) \cap X_{n-1}^{\prime} \subset L_{k}^{\prime} \cap t\left(L_{k}^{\prime}\right) \cap X_{n-1}=L_{k}^{\prime} \cap t\left(L_{k}\right) \cap X_{n-1} \subset$ $\left(X_{1} \backslash X_{n-1}\right) \cap X_{n-1}=\emptyset$.

In case $s(L)=L$, one can choose the function $\delta$ such that $\delta s=\delta$ so that $s(W)=W$. We forget all $L_{k}, k \geq 0$, consider instead only $L$ and note that since $s(L)=L$ there exists $n>0$ such that $L \cap t^{n}(L)=\emptyset$. We define analogously $X_{i}=L \cap \ldots \cap t^{i}(L)$ for $i \geq 0$. Since $s$ and $t$ commute, each $X_{i}, i \geq 0$, satisfies $s\left(X_{i}\right)=X_{i}$; therefore we can choose the family of arcs changing $L$ to $L^{\prime}$ so that $s\left(L^{\prime}\right)=L^{\prime}$.

4.5. Theorem. Let $H$ be a homeomorphism of the torus $\mathbf{T}^{2}$ preserving orientation which admits a lift $h$ to $\mathbf{R}^{2}$ without fixed point. Then there exists an essential simple closed curve on $\mathbf{T}^{2}$ which lifts to a Brouwer line of $h$.

Proof. Applying Lemma 3.6, we get a finite number of generalised critical discs $\left\{D_{1}, \ldots, D_{r}\right\}$ such that every critical disc centered on $[0,1] \times[0,1]$ admits one of the $D_{i}$ 's, $1 \leq i \leq r$ as a high strict extension. Since $h$ commutes with $s$ and $t$, we have in fact a finite set of Euclidean generalised critical discs $\left\{D_{1}, \ldots, D_{r}\right\}$ such that for every critical disc $D$ of $\mathbf{R}^{2}$ there exists $n, m \in \mathbf{Z}$ and $i$ between 1 and $r$ such that $s^{n} t^{m}\left(D_{i}\right)$ is a high strict extension of $D$.

We choose then a critical disc $D$. To this disc we associate a disc $D^{\prime}$ from $\left\{s^{n} t^{m}\left(D_{i}\right) \mid n, m \in \mathbf{Z}, 1 \leq i \leq r\right\}$ as just said. Since the underlying disc to $D^{\prime}$ is critical, there exists also $D^{\prime \prime}$ in the same set which is a high extension of $D^{\prime}$. In that way, we construct an infinite sequence $D^{\prime}, D^{\prime \prime}, \ldots, D^{(n)}, \ldots$ of generalised critical discs such that $\bigcup_{i=1}^{i=n} D^{(i)}$ is a critical topological disc for every $n$ (according to Lemma 3.5). Now, the finiteness of $\left\{D_{1}, \ldots, D_{r}\right\}$ implies that there exists $n, m \in \mathbf{Z}$ and $j>i \geq 1$ such that $D^{(j)}=s^{n} t^{m}\left(D^{(i)}\right)$. We note that

$$
C=\bigcup_{p \in \mathbf{Z}}\left(s^{n} t^{m}\right)^{p}\left(D^{(i)} \cup \ldots \cup D^{(j-1)}\right)
$$

is a critical band since $\left(s^{n} t^{m}\right)^{p+1}\left(D^{(i)}\right)=\left(s^{n} t^{m}\right)^{p}\left(D^{(j)}\right)$ is a high extension of $\left(s^{n} t^{m}\right)^{p}\left(D^{(j-1)}\right)$. Of course, $s^{n} t^{m}(C)=C$ and we can find a Brouwer line $L$ inside $C$ such that $s^{n} t^{m}(L)=L$. If $n$ and $m$ are relatively prime (or if $n=0$ and $m=1$ or if $m=0$ and $n=1$ ) we can conjugate $h$ by an element of $S L(2, \mathbf{Z})$ and suppose that $m=0, n=1$, that is, that $s(L)=L$. Lemma 4.3 gives then a Brouwer line $L^{\prime}$ such that $s\left(L^{\prime}\right)=L^{\prime}$ and $t\left(L^{\prime}\right) \cap L^{\prime}=\emptyset$, which therefore goes down to a simple closed curve in $\mathbf{R} \times S^{1}$ free under the map induced by $t$ and then to a simple closed curve in $\mathbf{T}^{2}$. In case $d=\operatorname{gcd}(m, n)>1$, we apply Lemma 4.2 to the Brouwer lines $L, T(L), \ldots, T^{d-1}(L)$ where $T=s^{\frac{n}{d}} t^{\frac{m}{d}}$ is a translation of $\mathbf{R}^{2}$ such that $T^{d}(C)=C$ since $s$ and $t$ commute (if $n=0$ or $m=0$ we let $d=|n|+|m|$ ). The Brouwer line given by this lemma ( $\mathrm{Fr} R$ there) is invariant by the translation $T$ and we are back to the preceding case.

4.6. Lemma. There is no Euclidean free or critical half plane if $h$ is a lift to $\mathbf{R}^{2}$ of a homeomorphism $H$ of $S^{1} \times \mathbf{R}=\mathbf{R} / \mathbf{Z} \times \mathbf{R}$ isotopic to the identity. 
Proof. Suppose first that the boundary of the half plane $D$ is not parallel to the $x$-axis. Then, given $z \in \mathbf{R}^{2}$, there exists $n \in \mathbf{Z}$ such that $t^{n}(z)$ and $t^{n}(h(z))$ are in $\operatorname{int} D$, but $t^{n}(h(z))=h\left(t^{n}(z)\right)$ and so $D$ is not free or critical.

If the boundary of the free or critical half plane $D$ is parallel to the $x$-axis, then $H(\pi(\operatorname{int} D)) \cap \pi(\operatorname{int} D)=\pi(h(\operatorname{int} D) \cap \operatorname{int} D)=\emptyset$ (the first equality follows from the $t$-invariance of $D)$. But $\pi(\operatorname{int} D)$ is a neighborhood of an end of $S^{1} \times \mathbf{R}$ and $H$ must preserve the ends, a contradiction.

4.7. Theorem. Let $H$ be a homeomorphism of the open annulus $S^{1} \times \mathbf{R}$ isotopic to the identity and with a fixed point free lift $h$ to $\mathbf{R}^{2}$. Then, there exists an essential simple closed curve in the annulus free under $H$ (and therefore lifting to a Brouwer line for $h$ in $\mathbf{R}^{2}$ ) or there exists a properly embedded line in the annulus going from one end to the other which lifts to a Brouwer line for $h$.

Proof. Applying Lemma 3.6 to $[0,1] \times[n, n+1] \subset \mathbf{R}^{2}$, we get a finite set of generalised critical discs $\left\{D_{1}^{n}, \ldots, D_{r_{n}}^{n}\right\}$ such that every critical disc centered on $[0,1] \times[n, n+1]$ admits one of the $D_{i}^{n}, 1 \leq i \leq r_{n}$ as a high strict extension and another one as a low strict extension. Since $h$ and $t$ commute, for every critical disc $D$ centered on the band $\mathbf{R} \times[n, n+1]$ there exists a $t^{k}\left(D_{i}^{n}\right), 1 \leq i \leq r_{n}, k \in \mathbf{Z}$, which is a high strict extension of $D$ and another one which is a low strict extension. We can suppose, by adding, if necessary, a finite number of critical discs to each family $\left\{D_{1}^{n}, \ldots, D_{r_{n}}^{n}\right\}$, that $\mathcal{D}=\bigcup_{n \in \mathbf{Z}}\left\{D_{1}^{n}, \ldots, D_{r_{n}}^{n}\right\}$ covers $[0,1] \times \mathbf{R}$ and so, that $\pi(\mathcal{D})$ covers $A$.

4.8. Fact. Only a finite number of discs of the family $\mathcal{D}$ can meet a given band $B_{n}=\mathbf{R} \times[n, n+1]$.

Proof. First remark that critical discs centered on $[0,1] \times \mathbf{R}$ with radius going to infinity have to go to infinity. Because, on the contrary, since the centers of these discs have to go to infinity, we would get a free or critical Euclidean half plane contrary to Lemma 4.6.

Suppose then that an infinite number of discs $D_{k}^{\prime}$ in $\mathcal{D}$ meets the band $B_{n}$. By definition $D_{k}^{\prime}$ meets also a critical disc $D_{k}$ centered on $[0,1] \times\left[n_{k}, n_{k}+1\right]$ where $n_{k} \rightarrow \pm \infty$. If the radius of $D_{k}$ is bounded, the radius of $D_{k}^{\prime}$ has to go to infinity, but if the radius of $D_{k}$ is not bounded, we can suppose (passing to a subsequence) that it goes to infinity and, using the preceding remark, we conclude that the radius of $D_{k}^{\prime}$ still has to go to infinity. Since translates of critical discs are still critical, we can get a sequence of critical discs which have their centers on $[0,1] \times \mathbf{R}$, meet $B_{n}$ and have a radius going to infinity: this implies the existence of a free or critical Euclidean half plane contrary to Lemma 4.6.

We then choose a critical disc $D$. There is a disc $D^{\prime} \in \bigcup_{k, n \in \mathbf{Z}} t^{k}\left(\left\{D_{1}^{n}, \ldots, D_{r_{n}}^{n}\right\}\right)$ which is a high strict extension of $D$. Then there exists $D^{\prime \prime}$ in the same set which is a high strict extension of $D^{\prime}$ and so on ad infinitum. One gets an infinite sequence $D^{\prime}, D^{\prime \prime}, \ldots, D^{(n)}, \ldots$ of generalised critical discs such that $D \cup\left(\bigcup_{i>0} D^{(i)}\right)$ is a proper critical half band (see Lemma 3.5). The same reasoning with low extensions leads to a proper critical band $D_{\infty}=\left(\bigcup_{i<0} D^{(i)}\right) \cup\left(\bigcup_{i>0} D^{(i)}\right)$ made of generalised critical discs.

We will consider three cases covering all possibilities for the relative positions of the band $D_{\infty}$ and the bands $B_{n}=\mathbf{R} \times[n, n+1]$. 
First case. There is an infinite number of the discs $D^{(n)}$ which meet a fixed band $B_{k}$.

As a consequence of Fact 4.8 above, there exist $k \in \mathbf{Z}$ and $j>i \geq 1$ such that $D^{(j)}=t^{k}\left(D^{(i)}\right)$ so that $t^{k(l+1)}\left(D^{(i)}\right)=t^{k l}\left(D^{(j)}\right)$ is a high extension of $t^{k l}\left(D^{(j-1)}\right)$ and consequently $\left.C=\bigcup_{l \in \mathbf{Z}} t^{k l}\left(D^{(i)}\right) \cup \ldots \cup D^{(j-1)}\right)$ is a critical band. Of course $t^{k}(C)=C$ and $C$ contains a Brouwer line $L$ such that $t^{k}(L)=L$. Applying Lemma 4.2 , we see that there exists an essential simple closed curve as in the theorem.

Second case. For all $n \in \mathbf{Z}$, the band $D_{\infty}$ contains a nonzero but finite number of $D^{(i)}$ which meet the band $B_{n}$.

Therefore there exists a Brouwer line $L$ which projects onto and properly on the $y$-axis and we conclude the proof using Lemma 4.3.

Third case. There exists an $n$ such that $D_{\infty}$ does not contain any disc meeting the band $B_{n}$. In other words, $D_{\infty}$ is contained in a Euclidean half plane with horizontal boundary.

We can suppose that, for every $n \in \mathbf{Z}, \bigcup_{i<0} D^{(i)}$ and $\bigcup_{i>0} D^{(i)}$ contain only a finite number of discs meeting $B_{n}$, for otherwise we are in the first case. We distinguish two subcases:

Either $t\left(D_{\infty}\right) \cap D_{\infty} \neq \emptyset$. Consider then a Brouwer line $L \subset D_{\infty}$ such that $t(L) \cap L \neq \emptyset$. An application of Lemma 4.2 to the family $\left(t^{n}(L)\right)_{n \in \mathbf{Z}}$ of Brouwer lines leads to an essential simple closed curve as required in the theorem.

Or $t\left(D_{\infty}\right) \cap D_{\infty}=\emptyset$. In that case, by Lemma $2.1, t^{n}\left(D_{\infty}\right) \cap D_{\infty}=\emptyset$ for all $n \neq 0$ and we can find a Brouwer line $L$ such that $t^{n}(L) \cap L=\emptyset$ for all $n \neq 0$. Such a line goes down in the annulus onto a free proper line going from one end to itself.

To conclude the proof of the theorem it is therefore enough to show the following.

4.9. Affirmation. In the preceding situation, suppose that any band $D_{\infty}$ constructed as above starting from any critical disc of the family $\mathcal{D}=\bigcup_{n}\left\{D_{1}^{n}, \ldots, D_{r_{n}}^{n}\right\}$ satisfies $t^{n}\left(D_{\infty}\right) \cap D_{\infty}=\emptyset$ for all $n \neq 0$. Then there exists in the annulus an essential simple closed curve free under $H$ or there exists a line in the annulus joining the two ends of the annulus and lifting to a Brouwer line for $h$.

Proof. We denote by $N$ and $S$ the ends of the annulus $A=\mathbf{R} / \mathbf{Z} \times \mathbf{R}$. Choose for each $D \in \mathcal{D}$ a band $D_{\infty}$ constructed as above. Given the hypothesis there are four types of generalized critical discs in $\mathcal{D}=\bigcup_{n}\left\{D_{1}^{n}, \ldots, D_{r_{n}}^{n}\right\}$ : either the band $D_{\infty}$ associated to such a disc verifies that $\pi\left(D_{\infty}\right)$ goes from $N$ to $N$ and, according to the separation or not of $H\left(D_{\infty}\right)$ and $S$ by $D_{\infty}$, we get the types $\rightarrow N$ and $\leftarrow N$, or $\pi\left(D_{\infty}\right)$ goes from $S$ to $S$ and we get the types $\leftarrow S$ and $\rightarrow S$.

- Let us suppose now that there exist discs $D \in \mathcal{D}$ of type $\rightarrow S$ or $\leftarrow S$ such that the $\pi(D)$ 's meet arbitrarly small neighborhoods of $N$. We choose then a sequence $\left(D_{n}\right)_{n \geq 1}$ of such discs such that $\pi\left(D_{n}\right)$ meets $\mathbf{R} / \mathbf{Z} \times[n,+\infty$ [. To each $D_{n}$ is associated a properly embedded band $\pi\left(D_{\infty}^{n}\right)$ in the annulus whose two ends converge towards the end $S$ of the annulus $A$. Let $F_{k}$ be the union over $n$ of the set of connected components of $\pi\left(D_{\infty}^{n} \cap(\mathbf{R} / \mathbf{Z} \times[-k, k])\right)$ which join $\mathbf{R} / \mathbf{Z} \times\{-k\}$ to $\mathbf{R} / \mathbf{Z} \times\{k\}$. The $F_{k}$ are finite sets by Fact 4.6 and every element of $F_{k+1}$ restricts to one of $F_{k}$. By the set-theoretical lemma below, there exists a band of $A$ going from $N$ to $S$, made from a $\mathbf{Z}$ indexed sequence of elements of $\pi(\mathcal{D})$, and every element of this sequence being a high extension of the preceding one. Therefore, this band is critical for $H$ and we get a properly embedded free line in the annulus $A$ joining $N$ and $S$ as desired. 
Of course, we get the same conclusion if we suppose that there exist $\operatorname{discs} D \in \mathcal{D}$ of type $\rightarrow N$ or $\leftarrow N$ such that the $\pi(D)$ meet arbitrarly small neighborhoods of $S$.

- Let us suppose that the two cases considered in the preceding point do not show up and, in particular, that all discs $\pi(D) \in \pi(\mathcal{D})$ with $D$ of type $\rightarrow N$ avoid a neighbohood $O_{S}$ of $S$ and that all those of type $\rightarrow S$ avoid a neighborhood $O_{N}$ of $N$.

Let us first remark that, if $D \in \mathcal{D}$ is of type $\rightarrow S$ (resp. $\rightarrow N$ ), every point of $\pi(D)$ converges towards $S$ (resp. $N$ ) under the action of $H$. Indeed, if $D_{\infty}$ is the critical band associated to $D$, then all $h^{n}(D)$ are separated from $N$ by $D_{\infty}$ and the set of points in the plane separated from $N$ by $D_{\infty}$ and with $y$-coordinate bounded below is bounded. But all points of the plane are wandering under $h$, so if $x \in D$, then the $y$-coordinate of $h^{n}(x)$ goes to $-\infty$ as $n \rightarrow+\infty$ and $H^{n}(\pi(x))$ goes towards $S$.

For a band $D_{\infty}$ let $\hat{D}_{\infty}$ be the closure of the component of $\mathbf{R}^{2} \backslash D_{\infty}$ which contains $h\left(D_{\infty}\right)$. Then let $E_{N}=\bigcup\left\{\pi\left(\hat{D}_{\infty}\right) \mid D\right.$ of type $\left.\rightarrow N\right\}$ and $E_{S}=\bigcup\left\{\pi\left(\hat{D}_{\infty}\right) \mid D\right.$ of type $\rightarrow S\}$ where $D_{\infty}$ is the band associated to $D$. These sets are closed according to the current hypothesis and are disjoint according to the above remark. Therefore there exists an essential closed curve in $A$ which avoids $E_{N}$ and $E_{S}$ and that one can cover by a finite number of elements of $\mathcal{D}$ (since we arranged that $\pi(\mathcal{D})$ covers $A)$. These elements are all of type $\leftarrow N$ or $\leftarrow S$ and therefore, according to the remark, all of the same type. Applying Lemma 4.2 to the Brouwer lines associated to these discs and to all their images under powers of $t$ gives an essential simple closed curve free under $H$ in the annulus.

This finishes the proof of the affirmation and of the theorem.

4.10. Lemma. Let $\left(F_{n}\right)_{n \geq 1}$ be a numerable sequence of nonempty finite sets and $R_{i} \subset F_{i} \times F_{i-1}$ a sequence of relations such that for all $f_{i} \in F_{i}$ there exist $f_{i-1} \in$ $F_{i-1}$ such that $\left(f_{i}, f_{i-1}\right) \in R_{i}$. Then there exists a sequence $\left(f_{n}\right)_{n \geq 1}$ such that $f_{i} \in F_{i}$ and $\left(f_{i}, f_{i-1}\right) \in R_{i}$ for all $i \geq 1$.

Proof. Endow each $F_{i}$ with the discrete topology. Then $\Pi_{i \geq 1} F_{i}$ is compact and, if $k \geq 1, A_{k}=\left\{\left(f_{1}, \ldots, f_{k}\right) \mid\left(f_{i}, f_{i-1}\right) \in R_{i}, 2 \leq i \leq k\right\} \times \Pi_{i>k} F_{i}$ is a nonempty closed subset of $\Pi_{i \geq 1} F_{i}$. Therefore $\bigcap_{k>0} A_{k} \neq \emptyset$.

\section{WINKELNKEMPER'S CONJECTURE}

In [12], Winkelnkemper proved the following extension of the Poincaré-Birkhoff theorem.

Theorem. Let $H$ be a homeomorphism of the closed annulus $S^{1} \times[0,1]$ homotopic to the identity and let $h$ be a lift of $H$ to $\mathbf{R} \times[0,1]$ such that $h$ is not conjugate to the translation $t:(x, y) \mapsto(x+1, y)$. Then $h$ has a fixed point or there exists in the annulus an essential simple closed curve free under $H$.

Since the hypothesis $h$ not conjugate to $t$ makes sense on the open annulus, he could end his note with the following conjecture. Let $H$ be an orientation preserving and area preserving homeomorphism of the 2-sphere $S^{2}$ with two distincts stable fixed points $N$ and $S$. Then, if $h$ is a lift of $H$ to the universal cover of $S^{2} \backslash\{N, S\}$ without fixed point, $h$ is conjugate to $t$. The following theorem is a slightly stronger version of Winkelnkemper's conjecture. 
Definition. A fixed point $p$ of a homeomorphism $H$ is stable if there is a basis $\left\{U_{n}\right\}_{n \in \mathbf{N}}$ of neighborhoods of $p$ such that $H\left(U_{n}\right)=U_{n}$ for all $n \in \mathbf{N}$.

Remarks. 1) If $H$ has no wandering point, for the fixed point $p$ to be stable, it is enough that there exists a basis $\left\{U_{n}\right\}_{n \in \mathbf{N}}$ of neighborhoods of $p$ such that $H\left(U_{n}\right) \subset$ $U_{n}$. Indeed, if $H(U) \subset U$ for some open set $U$, then $U \backslash H(\bar{U})$ is a wandering open set.

2) We can suppose each $U_{n}$ connected by replacing each $U_{n}$ by the connected component of $U_{n}$ which contains $p$.

5.1. Theorem. Let $H$ be an orientation preserving homeomorphism of $S^{2}$ without wandering point and with two distinct stable fixed points $N$ and $S$. Then, if $h$ is a lift of $H$ to the universal cover of $S^{2} \backslash\{N, S\}$ without fixed point, then $h$ is conjugate to $t$.

Proof. Let $L$ be a Brouwer line for $h$ which projects down to the interior of a simple arc $\gamma$ in $S^{2}$ going from $S$ to $N$ as given by Theorem 4.7. Define $W=\bigcap_{n \in \mathbf{Z}} D\left(h^{n}(L)\right)$ and $W^{\prime}=\bigcap_{n \in \mathbf{Z}} G\left(h^{n}(L)\right)$; these are $h$-invariant closed sets. To show that $h$ is conjugate to $t$ it is enough to show that $W$ and $W^{\prime}$ are empty. By exchanging $h$ and $h^{-1}$ we can restrict to considering $W$.

Let $\left\{U_{n}\right\}_{n \in \mathbf{N}}$ and $\left\{V_{n}\right\}_{n \in \mathbf{N}}$ be bases of connected invariant neighborhoods of $N$ and $S$ respectively. Suppose there exists $p \in W$ and choose $n$ such that $p$ belongs to the $h$-invariant closed set $B_{n}=\mathbf{R}^{2} \backslash \pi^{-1}\left(U_{n} \cup V_{n}\right)$. We will now imitate the Winkelnkemper argument: first, since $p \in W$, we have $h^{-i}(p) \in D(L) \cap B_{n}$ for all $i \geq$ 0 . Second, since $L$ is proper, we can find $k \in \mathbf{Z}$ such that $p \in G\left(t^{k}(L)\right)=t^{k}(G(L))$, which implies $h^{-i}(p) \in t^{k}\left(G\left(h^{-i}(L)\right)\right) \subset t^{k}(G(L))$ for all $i \geq 0$. Therefore $h^{-i}(p)$ lies in the bounded set $D(L) \cap t^{k}(G(L)) \cap B_{n}$ for all $i \geq 0$. This is a contradiction, since all points of the plane are wandering under the Brouwer homeomorphism $h$.

\section{ACKNOWLEDGEMENTS}

I would like to thank F. Le Roux for useful comments on a preliminary version of this paper and the referee for his very careful reading.

\section{REFERENCES}

[1] S. Addas-Zanata, Some extensions of the Poincaré-Birkhoff theorem to the cylinder and a remark on mappings of the torus homotopic to Dehn twists, Nonlinearity 18 (2005), 2243-2260. MR2164740 (2006h:37064)

[2] L.E.J. Brouwer, Beweis des ebenen Translationssatzes, Math. Ann., 72 (1912), 37-54. MR1511684

[3] F. Béguin, S. Crovisier, F. Le Roux, Pseudo-rotations of the open annulus, Bull. Braz. Math. Soc. 37 (2006), 275-306. MR2266384

[4] M. Bestvina, M. Handel, An area preserving homeomorphism of $T^{2}$ that is fixed point free but does not move any essential simple closed curve off itself, Ergod. Th. and Dynam. Sys., 12 (1992), 673-676. MR1200335 (94d:58119)

[5] J. Franks, Generalizations of the Poincaré-Birkhoff theorem, Ann. of Math., 128 (1988), 139-151. MR951509 (89m:54052)

[6] L. Guillou, Théorème de translation plane de Brouwer et généralisations du théorème de Poincaré-Birkhoff, Topology, 33 (1994), 331-351. MR1273787 (95h:55003)

[7] B. de Kérékjartó, Vorlesüngen uber Topologie, Springer, Berlin (1923).

[8] B. de Kérékjartó, The plane translation theorem of Brouwer and the last geometric theorem of Poincaré, Acta Sci. Math. Szeged, 4 (1928-29), 86-102. 
[9] P. Le Calvez, Une version feuilletée équivariante du théorème de translation de Brouwer, Publications IHES, 102 (2005), 1-98. MR2217051

[10] A. Sauzet, Application des décompositions libres à l'étude des homéomorphismes de surfaces, Ph.D. thesis, Université Paris-Nord (2001).

[11] H. Terasaka, Ein Beweis des Brouwerschen ebenen Translationssatzes, Japan J. of Math., 7 (1930), 61-69.

[12] H.E. Winkelnkemper, A generalisation of the Poincaré-Birkhoff theorem, Proc. AMS, 102 (1988), 1028-1030. MR934887 (89e:55006)

[13] G. Whyburn, E. Duda, Dynamic Topology, Springer Verlag, 1979. MR526764 (80e:54001)

Institut Fourier B.P. 74, Université Grenoble 1, Saint-Martin-D'Hères 38402 Cedex France

E-mail address: lguillou@ujf-grenoble.fr 